\title{
Arco aórtico: ramas y variaciones de diámetro interno en cadáveres frescos
}

\author{
Aortic arch: Branches and internal diameter variations in fresh corpses
}

\author{
Jorge I. Pareja-Pineda ${ }^{1,2 *}$ y Juan C. Quintana-Castillo ${ }^{3}$
}

${ }^{1}$ Grupo Regional Noroccidente de Patología Forense, Instituto Nacional de Medicina Legal y Ciencias Forenses; ${ }^{2}$ Facultad de Medicina, Universidad de Antioquia; ${ }^{3}$ Grupo INFETTARE, Facultad de Medicina, Universidad Cooperativa de Colombia. Medellín, Colombia

\section{Resumen}

Introducción: El conocimiento del patrón de las estructuras que emergen del arco aórtico es importante para las intervenciones en el cuello y la cabeza. Las variantes anatómicas y el área interna de estos vasos son cruciales para el enfoque de terapias endovasculares más seguras. Objetivo: Mostrar las variantes anatómicas y el área de los vasos que tiene dicha variación. Materiales y métodos: Se seleccionaron cuatrocientos cadáveres frescos, del Instituto Nacional de Medicina Legal y Ciencias Forenses de Medellín, con edades comprendidas entre los 18 y 40 años, sin lesiones en el tórax o el cuello, a los cuales se les evaluaron sus arcos aórticos. Todas las variantes se fijaron mediante registro fotográfico y las medidas se tomaron con un calibrador Mitutoyo. Conclusión: Este es el segundo trabajo sobre el tema llevado a cabo en Colombia y el primero en usar cadáveres frescos que se incorporaron en la medida que cumplían los criterios de inclusión.

Palabras clave: Cadáveres. Arco aórtico. Variación anatómica.

\section{Abstract}

Introduction: The knowledge of the pattern of the structures that emerge from the aortic arch is important for neck and head interventions mainly. The presence of anatomical variants and the internal area of these vessels is of great importance for the approach of safer endovascular therapies. Objective: To show the anatomical variants present and the area of the vessels that present such variation. Materials and methods: Four hundred fresh cadavers, aged between 18 and 40 years, without chest or neck injuries, were selected to evaluate their aortic arches, in the National Institute of Legal Medicine and Forensic Sciences of the city of Medellin, Colombia. All the variants were fixed photographically and the measurements taken with Mitutoyo calibrator. Conclusion: This is the second work on the subject carried out in our country and the first one using fresh cadavers and in a random way.

Key words: Corpses. Aortic arch. Anatomic variation.

\section{Correspondencia:}

*Jorge I. Pareja-Pineda

E-mail: jipareja @medicinalegal.gov.co
Fecha de recepción: 03-09-2018

Fecha de aceptación: 12-06-2020

DOI: 10.24875/RCCAR.M21000004
Disponible en internet: 19-03-2021

Rev Colomb Cardiol. 2021;28(1):18-23

www.rccardiologia.com 0120-5633 / @ 2020 Sociedad Colombiana de Cardiología y Cirugía Cardiovascular. Publicado por Permanyer. Este es un artículo open access bajo la licencia CC BY-NC-ND (http://creativecommons.org/licenses/by-nc-nd/4.0/). 


\section{Introducción}

El arco aórtico es una continuación curva de la aorta ascendente y se localiza en el mediastino superior. En todos los textos de anatomía se describen tres ramas que emergen, de manera constante, desde éste: el tronco braquiocefálico, la arteria carótida común izquierda y la arteria subclavia izquierda; posteriormente, el tronco braquiocefálico se subdivide en la arteria carótida común derecha y la arteria subclavia derecha. Las variantes anatómicas del arco aórtico han sido descritas desde las distancias entre sus ramas hasta el número de estas presentes en él ${ }^{1-4}$. Las variantes anatómicas obedecen a alteraciones en el desarrollo de determinados arcos branquiales aórticos durante el período embriológico ${ }^{5,6}$. Tales variaciones anatómicas de las ramas presentes en el arco aórtico, han sido descritas en diferentes poblaciones con diversas etnias, y su incidencia se estima entre el 5 al $35 \%$ \%

Se han realizado diferentes estudios tanto en cadáveres como en vivos, utilizando la visualización directa en cadáveres sometidos a disección, preservados con formaldehído y también en fresco $0^{10-14}$, mediante el uso de doppler ${ }^{15}$, angiografía ${ }^{1,16} \mathrm{y}$, en las últimas décadas, tomografía axial computarizada y resonancia magnética nuclear ${ }^{17-20}$.

El conocimiento de la disposición de las ramas aórticas, sus variaciones anatómicas y la frecuencia de su presentación en un grupo de individuos es importante para el abordaje quirúrgico y radiológico en terapias endovasculares del cuello y la cabeza, al igual que es pertinente para los anatomistas.

\section{Materiales y métodos}

Cuatrocientos cadáveres frescos, con edades entre 18 y 40 años de edad, sin distinción de sexo ni lesiones en cuello y tórax fueron escogidos para evaluar las ramas que emergían de sus arcos aórticos, durante la necropsia médico-legal, practicada en la sede del Instituto Nacional de Medicina Legal y Ciencias Forenses de Medellín, en el año 2015.

Los arcos aórticos fueron expuestos luego de retirar el pericardio y desbridar el tejido conectivo y otras estructuras anatómicas aledañas, y fueron observados in situ, para posteriormente ser disecados y evaluados en fresco, abiertos por su porción inferior con el fin de exponer los orificios donde se originaban sus ramas emergentes. Seguidamente, se hicieron dos mediciones del área interna de estos con un calibrador Mitutoyo; la primera fue realizada por los investigadores $y$

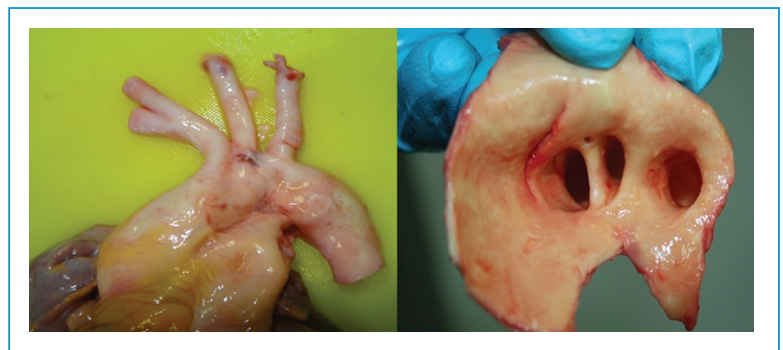

Figura 1. Tipo A (I).

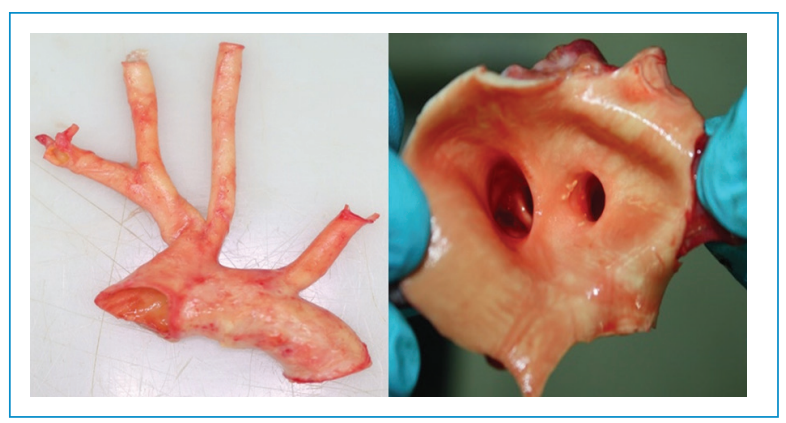

Figura 2. Tipo B (II).

la segunda por un par médico. Todas las estructuras encontradas fueron fijadas fotográficamente. Los patrones encontrados fueron separados de acuerdo con clasificación hecha por Adachi en 1928: tipo A (I), tipo B (II), tipo C (III) y tipo B (III) (Figs. 1-4).

Los valores obtenidos fueron consignados en una tabla de Excel. Los análisis estadísticos se realizaron en el programa PRISM versión 6 . En el análisis de los datos las variables continuas fueron expresadas como la media \pm desviación estándar $(D E)$, al igual que las variables nominales con sus proporciones.

\section{Resultados}

De los cuatrocientos cadáveres frescos (364 hombres y 36 mujeres), cuyos arcos aórticos fueron evaluados, todos eran de disposición hacia la izquierda, $316(79 \%)$, presentaban el patrón común de tres ramas -tronco braquiocefálico, arteria carótida común izquierda y arteria subclavia izquierda- y $84(21 \%)$ presentaron variantes anatómicas (un tronco común); es decir, un tronco que incluía el tronco braquiocefálico y, a su vez, la arteria subclavia izquierda en 14,25\% (57 cadáveres) una arteria vertebral izquierda en 4,75 \% (19 cadáveres) y una combinación entre tronco 
Tabla 1. Hallazgos de variantes anatómicas encontradas en el estudio de cadáveres

\begin{tabular}{|l|c|l|c|c|}
\hline Tipo de variante & Número de ramas & Descripción de ramas & n \\
\hline A & 3 & TBC, ACCl y ASCl & 316 & 79 \\
\hline B & 2 & TC y ASCl & 57 & 14.25 \\
\hline C & 4 & TBC, ACCI, AVI y ASCI & 19 & 4.75 \\
\hline D & 3 & TC, AVI y ASCI & 8 & 2 \\
\hline Total & - & - & 400 & 100 \\
\hline
\end{tabular}

ACCI: arteria carótida común izquierda; ASCl: arteria subclavia izquierda; TBC: tronco braquiocefálico; TC: tronco común; VI: arteria vertebral izquierda.

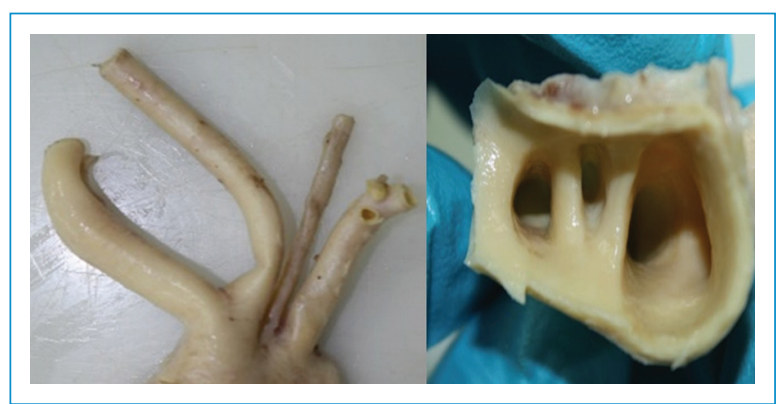

Figura 3. Tipo C (III).

común, arteria vertebral izquierda y arteria subclavia izquierda en un $2 \%$ (8 cadáveres). En este estudio no se encontraron otras variantes diferentes a las descritas en la literatura.

Los cuerpos evaluados tenían una edad promedio de 29,4 años ( $D E \pm 7.07$ ); 76 cadáveres $(90.5 \%$ ) fueron de sexo masculino y 8 fueron de sexo femenino $(9.5 \%)$ (Tabla 1).

La forma interna del tronco común fue oval o elíptica en 44 cadáveres ( $77.2 \%$ ) y esférica en 13 (22.8\%); la medida interna de los troncos elípticos fue de $15.2 \mathrm{~mm}$ $(D E \pm 2,33)$ en el horizontal y de $11.25 \mathrm{~mm}(D E \pm 0.18)$ en el vertical. Por su parte, los troncos comunes de forma circular tenían una medida interna de $12,6 \mathrm{~mm}$ (DE $\pm 2,63$ ).

La longitud del tronco común antes de dividirse en tronco braquiocefálico y arteria carótida común derecha fue de $10,79 \mathrm{~mm}(\mathrm{DE} \pm 2,34)$, en tanto que la medida interna del tronco braquiocefálico fue de $10.5 \mathrm{~mm}$ (DE \pm 1.6). La distancia entre el tronco común y la arteria carótida común izquierda fue de 2,09 mm (DE $\pm 1,09$ ). La medida interna del tronco braquiocefálico en los casos que no presentaban variante anatómica, fue de $14,3 \mathrm{~mm}(\mathrm{DE} \pm 1,1)$.



Figura 4. Otros (Tipo D).

El diámetro interno de la arteria carótida común izquierda fue de $5.65 \mathrm{~mm}(\mathrm{DE} \pm 1,4)$ y el de las arterias vertebrales, que emergían directamente del arco, fue de 3.2 ( $D E \pm 1,36)$. Todas alcanzaban el foramen transverso de la vértebra cervical $C \mathrm{~V}$; la distancia entre la arteria vertebral izquierda y la arteria subclavia izquierda fue de $1,38 \mathrm{~mm}(\mathrm{DE} \pm 1,46)$.

\section{Discusión}

El arco aórtico se desarrolla del saco aórtico, cuarto arco aórtico izquierdo, y otra parte del lado izquierdo del dorso de la aorta. Las variaciones están relacionadas con el proceso de fusión, así como con el proceso de absorción de algunos arcos al interior del saco aórtico durante la embriogénesis, lo cual lleva al incremento o decrecimiento del número de ramas emergentes ${ }^{5,7-21}$. Entre estas variantes anatómicas se encuentran, en el arco aórtico, -con mucha frecuencia según los estudios reportados- un tronco común donde, desde un ostium común, da origen al tronco braquiocefálico y a la arteria carótida común izquierda ${ }^{1,6-10,17-19,21}$. En este estudio constituyó el $14.25 \%$ de todas las variantes, en concordancia con valores reportados en la literatura por Jalili, Patil, Natsis y Herrera $22,8,1,12$, superiores a los 
Tabla 2. Resultados encontrados en estudios previos en cadáveres

\begin{tabular}{|l|l|c|c|c|}
\hline Autor & Población & $\mathbf{n}$ & Porcentaje de variantes anatómicas & Referencia \\
\hline Nayak, et al. (2006) & India & 56 & 8.6 & 17 \\
\hline Young, et al. (2008) & Corea & 25 & 16 & 10 \\
\hline Ogeng'0, et al. (2010) & África & 113 & 32.7 & 7 \\
\hline Bhattarai y Poudel (2010) & Nepal & 85 & 20 & 11 \\
\hline Herrera, et al. (2012) & Colombia & 122 & 28.7 & 12 \\
\hline Rekha y Senthilkumar (2013) & India & 100 & 7.28 & 14 \\
\hline El presente estudio (2016) & Colombia & 400 & 21 & \\
\hline
\end{tabular}

Tabla 3. Resultados de estudios realizados en pacientes a través de imagenología (arteriografías, TAC, RM)

\begin{tabular}{|l|l|l|l|c|}
\hline Autor & Población & $\mathbf{n}$ & Porcentaje de variantes anatómicas & Referencia \\
\hline Satyapal, et al. (2003) & India & 320 & 5.3 & 21 \\
\hline Natsis, et al. (2009) & Grecia & 633 & 17 & 1 \\
\hline Müller, et al. (2011) & Alemania & 2033 & 13.3 & 16 \\
\hline Ergun, et al. (2013) & Turquía & 1001 & 14.8 & 20 \\
\hline Jalali, et al. (2016) & Irán & 226 & 15.1 & 22 \\
\hline Mustafa, et al. (2017) & Jordania & 500 & 38.8 & 18 \\
\hline El presente estudio (2016) & Colombia & 400 & 21 & \\
\hline
\end{tabular}

reportados por Satyapal con $3.4 \%$, Piyavisetpat con $5.97 \%$ y Shin con $8 \%{ }^{21,9,10}$, pero muy inferior a los hallazgos de Mustafá con $31.2 \%$ y Ogeng'o con 25.7 $\% 18,7$. Llama la atención que en la revisión bibliográfica en los trabajos de Müller ${ }^{16}$ y Ergun $^{20}$ no se halló esta variante.

El arco aórtico da origen a tres ramas: el tronco braquiocefálico, la arteria carótida común izquierda y la arteria subclavia izquierda. Este estudio demostró que dicha disposición estuvo presente en el $79 \%$ de los cuerpos examinados, con un $21 \%$ restante que presentaba variables anatómicas. La literatura consultada corrobora que la presencia de tronco braquiocefálico, arteria carótida común izquierda y arteria subclavia izquierda son el patrón de mayor frecuencia, en las diferentes series, tanto en cadáveres por observación directa $^{7,10-14}$ como en pacientes, mediante estudios radiológicos ${ }^{1,16,18,21}$. El $21 \%$ de las variantes encontradas en este estudio presenta similitud con las encontradas en el estudio de Herrera et al..$^{12}$, realizado con cadáveres en Bucaramanga, y con las exploraciones de Bhattarai y Poudel en Nepal ${ }^{11}$, pero se observa que dicho porcentaje se encuentra más alto que en los restantes grupos asiáticos y es muy inferior al del trabajo realizado por Ogeng'o ${ }^{7}$ (Tabla 2). Para el caso en el que se tomaron medidas a través de procedimientos imagenológicos ${ }^{1,16,18,20-22}$, se encontró mayor número de muestra, lo cual se explica debido a la facilidad de la obtención de esta y al tipo de estudio. Nuestros resultados muestran un porcentaje de variación anatómica que solo es superado por el trabajo de Mustafa et al. para la población de Jordania ${ }^{18}$ (Tabla 3 ) y fue mayor en el resto de los casos, siendo particularmente más alto respecto al trabajo de Satyapal et al. ${ }^{21}$, que obtuvo un porcentaje del $5.3 \%$.

La segunda variante más frecuente en el presente estudio, correspondió a la arteria vertebral izquierda, emergiendo directamente del arco aórtico entre la arteria carótida común izquierda y la arteria subclavia izquierda (y el tronco braquiocefálico en su posición habitual, con un porcentaje del $4.75 \%$. Los resultados reportados por Herrera, Shin y Patil $12,10,8$ fueron muy superiores, 8.2, 8 y $8 \%$ respectivamente, mientras que en los trabajos de Rekha, Piyavisetpat, Müller y Ergun ${ }^{14,9,16,20}$ los valores 
encontrados para esta variante fueron muy similares a los reportados en el presente trabajo, mientras que los de Natsis y Jalil ${ }^{1}, 22$ fueron extremadamente inferiores, $0.79 \%$ y $0.9 \%$, respectivamente.

La variante encontrada y denominada tronco común con presencia de la arteria vertebral izquierda, emergiendo directamente del arco aórtico, tercera variante encontrada, es una variante poco descrita en los estudios, con una presencia, según Celikyay del $0.4 \%{ }^{19}$, Rekha del $0.9 \%{ }^{14}$ y Jakanini del $17 \%{ }^{23}$. En el presente estudio fue del $2 \%$, dato similar a lo expuesto por Ogeng'o, con un $1.8 \%^{7}$, y dicha variante no aparece descrita en el estudio de Herrera ${ }^{12}$.

Respecto a las medidas internas encontradas en las variantes anatómicas, es poca la literatura que se encarga de esta descripción. Herrera ${ }^{12}$ describe un diámetro externo, en cadáveres frescos y preparados anatómicos, para el tronco braquiocefálico de $9.7 \mathrm{~mm}$ ( $\mathrm{DE} \pm 1.61$ ), para el tronco común de $14.3 \mathrm{~mm}$, para la arteria carótida común izquierda de $6.3 \mathrm{~mm}(\mathrm{DE} \pm 0.75)$, para la arteria vertebral izquierda mayor de $3.9 \mathrm{~mm}$ y para la distancia entre la arteria vertebral izquierda y la arteria subclavia izquierda de $1.75 \mathrm{~mm}(\mathrm{DE} \pm 0.64)$.

Alsaif ${ }^{24}$, en un estudio con cadáveres preservados con formalina, refiere un tronco braquiocefálico de $17.97 \mathrm{~mm}(\mathrm{DE} \pm 3.85)$, de la arteria carótida común izquierda de $9.77 \mathrm{~mm}(\mathrm{DE} \pm 1.91)$ y de la arteria vertebral izquierda de $5.5 \mathrm{~mm}$ e incluye la longitud del tronco común de $15 \mathrm{~mm}(\mathrm{DE} \pm 5.86)$ con un diámetro de $30.33 \mathrm{~mm}$. Yeri ${ }^{25}$, por su parte, en cadáveres fijados con formalina, encontró un tronco braquiocefálico de $20 \mathrm{~mm}$, una arteria carótida común izquierda de 14 $\mathrm{mm}$, una arteria subclavia izquierda de 17.9 y un tronco común de $2.6 \mathrm{~mm}$. Shin ${ }^{10}$, también en cadáveres preservados, describe un diámetro del tronco braquiocefálico de $18.3 \mathrm{~mm}$, de la arteria carótida común izquierda de $9.5 \mathrm{~mm}$ y de la arteria subclavia izquierda de $10.6 \mathrm{~mm}$.

Macchi $^{13}$, mediante doppler, obtuvo un tronco braquiocefálico de $6.31 \mathrm{~mm}(\mathrm{DE} \pm 0.87$ ), la arteria carótida común izquierda $7.37 \mathrm{~mm}(\mathrm{DE} \pm 0.47)$ y la arteria vertebral izquierda $3.32 \mathrm{~mm}(\mathrm{DE} \pm 0.59)$. Kahraman ${ }^{15}$, en un trabajo con pacientes mediante arteriografía, señala un tronco braquiocefálico de $12.07 \mathrm{~mm}(\mathrm{DE} \pm 1.58)$, arteria carótida común izquierda de $7.15 \mathrm{~mm}$ y arteria subclavia izquierda de $9.04 \mathrm{~mm}(\mathrm{DE} \pm 1.74)$. Finalmente, Matula ${ }^{26}$, a través de preparados anatómicos, pacientes con angiografías y estudios doppler, encontró que el diámetro de la arteria vertebral izquierda era menor de $3.5 \mathrm{~mm}$.
En cuanto al presente trabajo, se encontró que la medida interna del tronco braquiocefálico en los casos que no presentaban variantes anatómicas fue de 14,3 mm (DE 1,1), muy superior al descrito por Macchi $^{13}$ y Herrera ${ }^{12}$, pero inferior a los hallazgos de Yerii ${ }^{25}$.

La longitud del tronco común fue de 10,79 mm (DE $\pm 2,34$ ) y la medida interna del tronco braquiocefálico fue de $10.5 \mathrm{~mm}(\mathrm{DE} \pm 1.6)$, la cual fue inferior a los hallazgos encontrados por Alsaif, $15 \mathrm{~mm}$ y $30.33 \mathrm{~mm}^{24}$, respectivamente, y muy similar al descrito por Kodama, $11 \mathrm{~mm}^{27}$. Es importante resaltar que ninguno de los estudios revisados refiere la distancia entre el tronco braquiocefálico y la arteria carótida común izquierda, que se desprenden de un mismo ostium, y que para nuestro caso fue de 2,09 mm (DE $\pm 1,09$ ).

El diámetro interno de la arteria carótida común izquierda fue de $5.65 \mathrm{~mm}(\mathrm{DE} \pm 1,4)$, muy inferior a lo relatado por Yeri, Shin, Kahraman y Alsaif 25,10,15,24.

En cuanto a las arterias vertebrales, acorde con lo relatado en la literatura, se ha descrito que el diámetro interno de una arteria vertebral menor de $3.5 \mathrm{~mm}$ se considera hipoplásica ${ }^{28}$. Para el caso nuestro, el promedio fue de $3.2 \mathrm{~mm}(\mathrm{DE} 1,36)$ y la distancia entre la arteria vertebral izquierda y la arteria subclavia izquierda fue de $1,38 \mathrm{~mm}(\mathrm{DE} \pm 1,46)$; esta última fue muy similar a la encontrada por Herrera ${ }^{13}, 1,75 \mathrm{~mm}$ ( $D E \pm 0.64)$, pero para este mismo autor el hallazgo del diámetro fue de $3.9 \mathrm{~mm}$. Matula ${ }^{26}$, por su parte, encontró que un alto porcentaje de arteria vertebral izquierda fue inferior a $3.5 \mathrm{~mm}$; Macchi ${ }^{13}$ reportó una medida de $3.32 \mathrm{~mm}$ (DE 0.59), mientras que Alsaif ${ }^{24}$ describió una medida interna de la arteria vertebral izquierda de $5.5 \mathrm{~mm}$.

Muchos de los trabajos publicados a la fecha se han enfocado en muestras poblacionales, como griegos ${ }^{1}$, indios $^{8,13}$, nepaleses ${ }^{11}$, coreanos $^{10}$, keniatas ${ }^{7}$ y jordanos $^{18}$. En nuestro caso, debido al proceso de mestizaje, se considera mejor hacer alusión a una muestra en determinada población y no hacerla extensiva a toda la población colombiana y, más bien, promover otros estudios en diferentes ciudades del país con el ánimo de unificar cifras.

Otro de los puntos a considerar, en cuanto a las discrepancias, en las medidas encontradas de las estructuras del arco aórtico donde aparecían variantes anatómicas, frente a los distintos autores, son las muestras y métodos utilizados para tal proceso; autores como Ogeng'o, Shin, Bhattarai, Herrera, Rekha y Nayak $7,10,11,12,14,17$ utilizaron material preservado mediante formaldehído, el cual produce alteraciones en 
los especímenes anatómicos. Por otro lado, los estudios radiológicos desarrollados por Natsis, Müller, Ergun, Mustafa, Satyapal y Jalili1,16,20,18,21,22, tomografía computarizada, arteriografías, doppler y resonancia magnética, realizados en pacientes que asistieron a consulta por trastornos relacionados con el sistema circulatorio, dan unas mediciones aproximadas, más si se tiene en cuenta que dichas valoraciones fueron realizadas posteriormente y no al momento del examen diagnóstico.

\section{Conclusión}

Es importante señalar que el porcentaje de variantes encontradas en este estudio, aunque ligeramente inferior a las encontradas por Herrera ${ }^{12}$, -el primer estudio de este tipo realizado a este respecto-, no difiere en grandes proporciones y es una referencia para cirujanos y otras especialidades médicas relacionadas con enfermedades y terapia endovascular en la cabeza y el cuello que permitirán un diagnóstico adecuado y predecir una serie de complicaciones con la aplicación de procedimientos más seguros.

La realización de este tipo de estudios en cadáveres frescos permite una aproximación más cercana a la realidad respecto a las mediciones de las estructuras vasculares, que en las muestras de cadáver conservadas con formaldehído y utilizadas en salas de autopsias están sujetas a distorsiones por efecto químico y manipulación por estudiantes y funcionarios de estos laboratorios.

\section{Conflicto de intereses}

Los autores declaran que no existe conflicto de intereses.

\section{Responsabilidades éticas}

Protección de personas y animales. Los autores declaran que para esta investigación no se han realizado experimentos en seres humanos ni en animales.

Confidencialidad de los datos. Los autores declaran que han seguido los protocolos de su centro de trabajo sobre la publicación de datos de pacientes.

Derecho a la privacidad y consentimiento informado. Los autores declaran que en este artículo no aparecen datos de pacientes.

\section{Bibliografía}

1. Natsis $\mathrm{KI}$, Tsitouridis IA, Didagelos MV, Fillipidis AA, Vlasis KG, Tsikaras PD. Anatomical variations in the branches of the human aortic arch in 633 angiographies: clinical significance and literature review. Surg Radiol Anat. 2009;31(5):319-23.

2. Bannister WWD. Gray's Anatomy $37^{\text {th }}$ ed., International Edition. London: Churchill Livingstone; 1989.

3. Gardner ED, O'Rahilly R. Anatomy: a regional study of human structure: W.B. Saunders; 1986.

4. Adachi B, Hasebe K, Igakubu KD. Das Arteriensystem der Japaner: Kenkyusha; 1928.

5. Sadler TW. Cardiovascular system. Langman's Medical Embryology: Lippincott Williams \& Wilkins; 2006.

6. Moore KL, Persaud TVN, Torchia MG. Muscular System. The Developing Human: Clinically Oriented Embryology. Cap 15. Philadelphia. Elsevier Health Sciences; 2015.

7. Ogeng'o J, Olabu B, Gatonga P, Munguti J. Branching pattern of aortic arch in a kenyan population. J Morphol Sci. 2010;27(2):51-5.

8. Patil ST, Meshram MM, Kamdi NY, Kasote AP, Parchand MP. Study on branching pattern of aortic arch in Indian. Anat Cell Biol. 2012;45(3):203-6.

9. Piyavisetpat $N$, haksinawisut $P$, Tumkosit $M$. Aortic arch branches' variations detected on chest CT. Asian Biomed (Res Rev News). 2011;5(6):817-23.

10. Shin IY, Chung YG, Shin WH, Im SB, Hwang SC, Kim BT. A morphometric study on cadaveric aortic arch and its major branches in 25 korean adults: the perspective of endovascular surgery. J Korean Neurosurg Soc. 2008;44(2):78-83

11. Bhattarai C, Poudel PP. Study on the variation of branching pattern of arch of aorta in Nepalese. Nepal Med Coll J. 2010;12(2):84-6.

12. Herrera N, Ballesteros LE, Forero PL. Characterization of aortic arch branches in a Colombian population sample. A study with autopsy material. Int J Morphol. 2012;30(1):49-55.

13. Macchi $\mathrm{C}$, Giannelli $\mathrm{F}$, Catini $\mathrm{C}$. The measurement of the calibers of the branches of the aortic arch: a statistical investigation of 430 living subjects using ultrasonic tomography. Italian journal of anatomy and embryology. Archivio Italiano di Anatomia ed Embriologia. 1993:98(2):69-79.

14. Rekha P, Senthilkumar S. A study on branching pattern of human aortic arch and its variations in south indian population. J Morphol Sci. 2013;30(1):11-5.

15. Kahraman H, Ozaydin M, Varol E, Aslan SM, Dogan A, Altinbas A, et al. The diameters of the aorta and its major branches in patients with isolated coronary artery ectasia. Texas Heart Institute Journal. 2006;33(4):463-8.

16. Muller M, Schmitz BL, Pauls S, Schick M, Rohrer S, Kapapa T, et al. Variations of the aortic arch - a study on the most common branching patterns. Acta Radiol. 2011;52(7):738-42.

17. Nayak SR, Pai MM, Prabhu LV, Costa S, Shetty P. Anatomical organization of aortic arch variations in the India: embryological basis and review. Jornal Vascular Brasileiro. 2006;5(2):95-100.

18. Mustafa AG, Allouh MZ, Ghaida JH, Al-Omari MH, Mahmoud WA. Branching patterns of the aortic arch: a computed tomography angiography-based study. Surg Radiol Anat. 2017;39(3):235-42.

19. Celikyay ZR, Koner AE, Celikyay F, Deniz C, Acu B, Firat MM. Frequency and imaging findings of variations in human aortic arch anatomy based on multidetector computed tomography data. Clin Imaging. 2013;37(6):1011-9.

20. Ergun E, Simsek B, Kosar PN, Yilmaz BK, Turgut AT. Anatomical variations in branching pattern of arcus aorta: 64-slice CTA appearance. Surg Radiol Anat. 2013;35(6):503-9.

21. Satyapal KS, Singaram S, Partab P, Kalideen JM, Robbs JV. Aortic arch branch variations--case report and arteriographic analysis. South African Journal of Surgery Suid-Afrikaanse Tydskrif Vir Chirurgie. 2003;41(2):48-50.

22. Jalali Kondori B, Asadi MH, Rahimian E, Tahsini MR. Anatomical Variations in Aortic Arch Branching Pattern. Arch Iran Med. 2016;19(1):72-4.

23. Jakanani GC, Adair W. Frequency of variations in aortic arch anatomy depicted on multidetector CT. Clin Radiol. 2010;65(6):481-7.

24. Alsaif HA, Ramadan WS. An anatomical study of the aortic arch variations. JKAU Med Sci. 2010;17:37-54.

25. Yeri LA, Gómez JE. Yeri LA, X, Variation of the Origin of Aortic Arch Branches: In Relationship with Plates of Atheroma. Int $\mathrm{J}$ Morphol. 2011;29(1):182-6.

26. Matula C, Trattnig S, Tschabitscher M, Day JD, Koos WT. The course of the prevertebral segment of the vertebral artery: anatomy and clinical significance. Surg Neurol. 1997;48(2):125-31.

27. Kodama J, Tsuno K, Sekine G, Ohmori T, Toh H, Takei T, et al. Anomalous case of the Leith common carotid artery arising from the brachiocephalic trunk. Fukuoka Shika Daigaku Gakkai Zasshi. 1989;16:609-13.

28. Paturet G. Traité d'anatomie humaine: pt. 1. Appareil circulatoire (a l'exclusion des veines). Paris: Masson; 1964. 